\title{
Feasibility of antiretroviral treatment monitoring in the era of decentralized HIV care: a systematic review
}

\author{
Minh D. Pham ${ }^{1,2}$, Lorena Romero ${ }^{3}$, Bruce Parnell ${ }^{1}$, David A. Anderson ${ }^{1,4}$, Suzanne M. Crowe \\ and Stanley Luchters ${ }^{1,2,7^{*}}$
}

\begin{abstract}
Background: Regular monitoring of HIV patients who are receiving antiretroviral therapy (ART) is required to ensure patient benefits and the long-term effectiveness and sustainability of ART programs. Prompted by WHO recommendations for expansion and decentralization of HIV treatment and care in low and middle income countries, we conducted a systematic review to assess the feasibility of treatment monitoring in these settings.
\end{abstract}

Methods: A comprehensive search strategy was developed using a combination of MeSH and free text terms relevant to HIV treatment and care, health service delivery, health service accessibility, decentralization and other relevant terms. Five electronic databases and two conference websites were searched to identify relevant studies conducted in LMICs, published in English between Jan 2006 and Dec 2015. Outcomes of interest included the proportion of patients who received treatment monitoring and health system factors related to monitoring of patients on ART under decentralized HIV service delivery models.

Results: From 5363 records retrieved, twenty studies were included in the review; all but one was conducted in subSaharan African countries. The majority of studies (15/20) had relatively short follow-up duration ( $\leq 24$ months), and only two studies were specifically designed to assess treatment monitoring practices. The most frequently studied follow-up period was 12 months and a wide range of treatment monitoring coverage was observed. The reported proportions of patients on ART who received CD4 monitoring ranged from very low $(6 \% ; N=2145)$ to very high (95\%; $\mathrm{N}=488$ ). The median uptake of viral load monitoring was $86 \%$ with studies in program settings reporting coverage as low as $14 \%$. Overall, the longer the follow-up period, the lower the proportion of patients who received regular monitoring tests; and programs in rural areas reported low coverage of laboratory monitoring. Moreover, uptake in the context of research had significantly better where monitoring was done by dedicated research staff. In the absence of point of care (POC) testing, the limited capacity for blood sample transportation between clinic and laboratory and poor quality of nursing staff were identified as a major barrier for treatment monitoring practice.

Conclusions: There is a paucity of data on the uptake of treatment monitoring, particularly with longer-term followup. Wide variation in access to both virological and immunological regular monitoring was observed, with some clinics in well-resourced settings supported by external donors achieving high coverage. The feasibility of treatment monitoring, particularly in decentralized settings of HIV treatment and care may thus be of concern and requires further study. Significant investment in POC diagnostic technologies and, improving the quality of and training for nursing staff is required to ensure effective scale up of ART programs towards the targets of 90-90-90 by the year 2020.

Keywords: HIV, Decentralized care, Task-shifting, Antiretroviral treatment, Treatment monitoring, Viral load, CD4, Systematic review

\footnotetext{
*Correspondence: stanley.luchters@burnet.edu.au

${ }^{1}$ Burnet Institute, 85 Commercial Road, Melbourne, VIC 3004, Australia

Full list of author information is available at the end of the article
} 


\section{Background}

Increasing access to antiretroviral therapy (ART) for people living with HIV/AIDS has been identified as a key strategy to curb the HIV epidemic and avoid its cost in the future [1]. In 2015, an estimated 15 million people living with HIV/AIDS (PLWHs) were receiving ART, a remarkable milestone in the fight against HIV/AIDS [2]. However, in order to achieve the ambitious sustainable development goal of ending the HIV epidemic by 2030, greater efforts are required in expanding ART coverage and improving quality of services with innovative and effective service delivery models.

In a number of the low and middle income countries (LMICs) most affected by the epidemic, decentralization of HIV treatment and care, linked with task-shifting, has been implemented in response to the need for scaling up service provision [3]. Evidence from existing systematic reviews suggests that relocation of ART services closer to patients' homes through decentralized care can improve patient access and adherence to HIV treatment with noninferior quality of care as compared to centralized, hospital-based care [4-6].

Current WHO guidelines on the use of ARV drugs for HIV treatment and prevention strongly recommend virological monitoring as the strategy of choice for monitoring responses to ART [7]. Immunological monitoring (CD4 testing) is being scaled back for assessment of treatment responses where VL testing is available, but will still be required for the foreseeable future in many settings to determine the level of HIV-induced immune deficiency, including the need for screening and prophylaxis for serious co-infections, and to prioritize initiation of HIV treatment. Clinical monitoring is essential for all patients who are receiving ART to monitor patient responses to treatment and diagnose potential treatment failure [8]. In addition, monitoring of ARV drug toxicity is recommended, as delaying drug substitutions when there are adverse drug effects may not only cause harm but also result in non-adherence leading to drug resistance and treatment failure. The latter will compromise the effectiveness of available ART regimens, increase spread of drug-resistant HIV, increase HIV incidence, morbidity and mortality and negatively impact the long-term sustainability and efficacy of ART programs in LMICs.

Given the current limited health system capacity in many LMICs, meeting WHO's recommendations regarding regular monitoring of patients' responses to treatment, including monitoring of drug toxicity, may pose major challenges to the health system with possible negative impacts on quality and sustainability of HIV services in the future $[9,10]$. This vulnerable situation is particularly likely while rapid scale up of decentralized provision of ART is being prioritized.
This systematic review assessed the feasibility of ART treatment monitoring in settings of decentralized HIV treatment and care in LMICs.

\section{Methods \\ Literature search strategy}

The preferred reporting item for systematic reviews and meta-analysis (PRISMA statement) [11] was used to guide the conduct of this review. A literature search strategy was developed to identify relevant studies that involve decentralization of HIV treatment and care in low and middle income countries, published in English between Jan 2006 and Dec 2015. Key search terms include MeSH and free text terms relevant to HIV infection, HIV treatment and care, health service delivery models and service accessibility such as: "HIV", "HIV infection", "Antiretroviral therapy", "ART", "HAART", "delivery of health care" "primary health care", "community health services", "home-based", "decentral", "task-shift". Search terms also included those that refer to treatment monitoring including "treatment outcomes", "adverse effect" and "toxicity". The search strategy was first conducted in Medline (see Additional file 1: Annex S1), then adapted to run across CENTRAL, CINAHL, EMBASE, Scopus and Web of science. Conference abstracts were also searched from International AIDS Society and CROI conference websites. Grey literature resources and reference lists of existing systematic reviews were searched to identify relevant studies. For the purpose of this review, "feasibility" is defined as capacity of health system to provide and patient's accessibility to ART monitoring services following WHO's recommendations [7].

\section{Study selection}

Studies met inclusion criteria for this review if they: (i) involved HIV infected patients requiring ART and treatment follow-up, and/or healthcare workers involved in providing ART services; (ii) involved a decentralized model of HIV treatment and care which was defined as ART initiation and/or ART monitoring services provided at non-hospital settings: primary health facility or community level (through home-based delivery or community outreach including mobile health services); and (iii) reported one or more of the primary outcomes of interest as defined below.

1. Proportion of patients receiving (with data documented) CD4 count, clinical HIV staging, and/or HIV viral load monitoring at treatment follow-up at regular intervals (6 or 12 months);

2. Proportion of patients receiving ARV drug toxicity monitoring (clinical and/or biomedical) at treatment follow-up at regular intervals; and/or 
3. Reported enablers, barriers and other implementing issues related to monitoring of ART services, including any of the following (a) human resources (availability and quality of clinical staff; staff competency training); (b) availability of, and access to, clinical, biochemical monitoring tools for monitoring treatment response, diagnosing ARV drug toxicity, and/ or treatment failure; (c) supply chain management: reagents, equipment maintenance, etc. under decentralized HIV care; (d) patient and provider's attitude towards decentralization of HIV treatment and care.

Secondary outcomes included: (1) Proportion of patients with reported treatment failure, and (2) Proportion of patients who switched to a second line ARV drug.

In order to be eligible for inclusion, studies must have been conducted in LMICs and have reported at least one primary outcome or provided data which allowed for calculation of treatment monitoring uptake.

\section{Data extraction and data synthesis}

Data were extracted electronically using a pre-constructed, standardized data extraction form. Double data extraction with $20 \%$ duplication was performed by two independent reviewers. Extracted information included: study details (author/year, objective, design, number of patient enrolled), study population criteria, mode of ART services and outcome of interest. Data on outcomes of interest were grouped, presented and compared by models of service delivery (decentralized vs centralized), time point of treatment follow-up, and study design/study setting context. Quantitative data were presented and analyzed descriptively and data across studies were pooled, provided study interventions and populations were sufficiently similar. Qualitative data were thematically categorized using main themes relevant to the research questions, which emerged from data extracted.

\section{Results}

\section{Study characteristics}

The search strategy identified 5363 titles after duplicates were removed. Screening of titles plus abstracts with exclusion of clearly irrelevant studies resulted in 58 eligible studies for full text review, of which 20 studies (19 articles and one abstract [12]) met all of the inclusion criteria, and were included in the review (Fig. 1).

All but one of these 20 studies were conducted in sub-Saharan Africa (SSA): 10 studies were from various urban, peri-urban and rural settings in South Africa (SA), one study was from rural and urban Ethiopia, one from rural Lesotho, one from rural and urban Kenya, two from rural Rwanda, one from urban Mozambique, one from rural Zimbabwe, two from rural Swaziland, and one from Asia (Thailand). Only two studies were specifically designed to assess the coverage of HIV treatment monitoring services in a decentralized setting; other studies evaluated and reported treatment outcomes. Only one study reported the proportion of patients who developed drug toxicity and two studies provided qualitative data (Table 1).

\section{HIV viral load (VL) monitoring}

Twelve studies (Table 2) provided data regarding the proportion of patients who received regular VL monitoring, among which 11 studies reported the proportion of patients receiving VL monitoring at 12 months followup, with a median service uptake of $86 \%$. The highest coverage of virological monitoring services was reported from two randomized control trials (RCT) conducted in SA [13] and Kenya [14] with 92\% (2582/2823) and 99\% $(86 / 87)$ uptake; both studies were conducted by dedicated research staff who were not part of the routine clinical service. The lowest reported proportion of patients with VL monitoring data came from a retrospective cohort study conducted between 2002 and 2008 in rural Thailand [15] with only $14.3 \%(22 / 154)$ of patients having VL data available at baseline and at least one treatment follow-up 12-48 months after treatment initiation. The authors reported that routine VL testing was not available, baseline VL data were available only for a subset of the study participants and VL was determined at 12 months intervals during the 48 months of study.

In four studies that reported the proportion of patients who received VL monitoring in both centralized and decentralized models of care, two studies reported a higher proportion of monitoring of patients attending centralized care (vs decentralized care): $99 \%$ (1774/1958) versus $91 \%(676 / 681)$ [16] and $29 \%$ (38/133) versus $14 \%$ (22/154) [15], while another two studies reported a similar or higher proportion of patients with access to VL monitoring with decentralized care (vs centralized care): $92 \%$ versus $90 \%$ [13] and $61 \%$ (296/482) versus $14 \%$ $(41 / 289)$ [17]. In the two latter studies, both conducted in SA, the difference in service coverage between models of care was not discussed; however, one study [13] reported results of a 30 month randomized trial aimed to assess the effects of an outreach training program provided to nurses for ART initiation and prescribing at primary care clinics while the other [17] reported outcomes of a community-based, decentralized HIV services delivery program supported by Medecins Sans Frontieres (MSF).

Overall, studies conducted in urban settings reported a higher uptake of VL monitoring services: three studies conducted in urban HIV clinic settings in SA $[16,18,19]$ reported more than $80 \%$ of patients had VL data available after 6-24 months on treatment while three other studies 

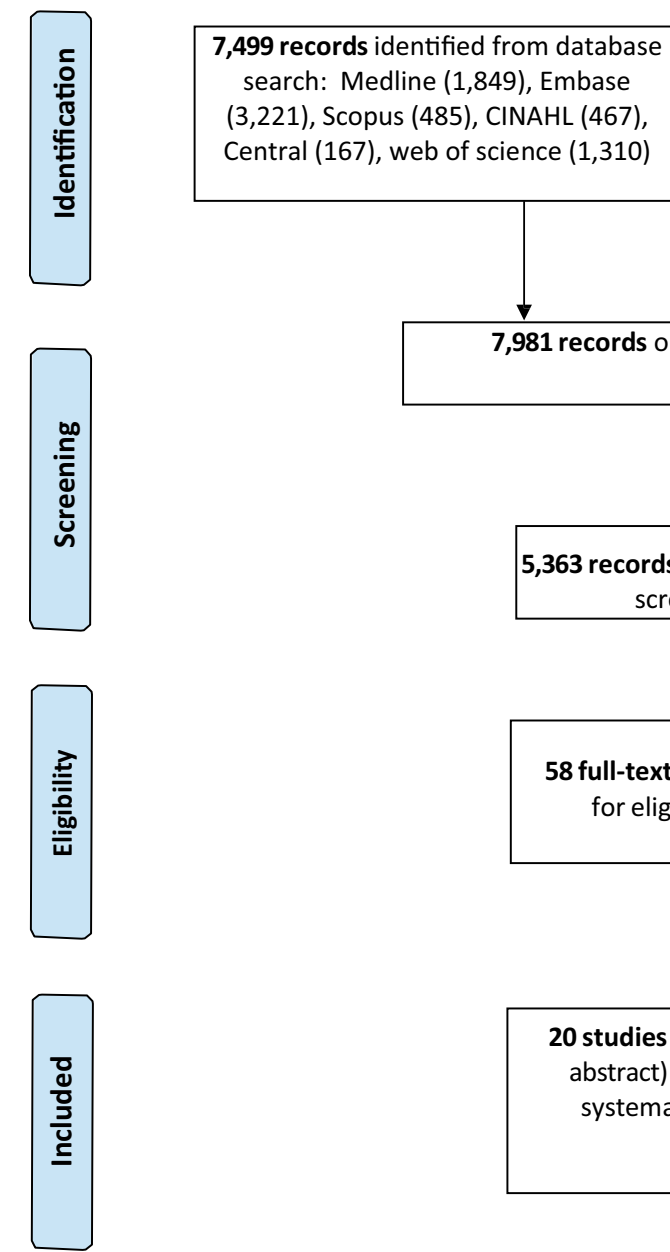

482 additional records identified

through other sources (conference websites, grey literature)

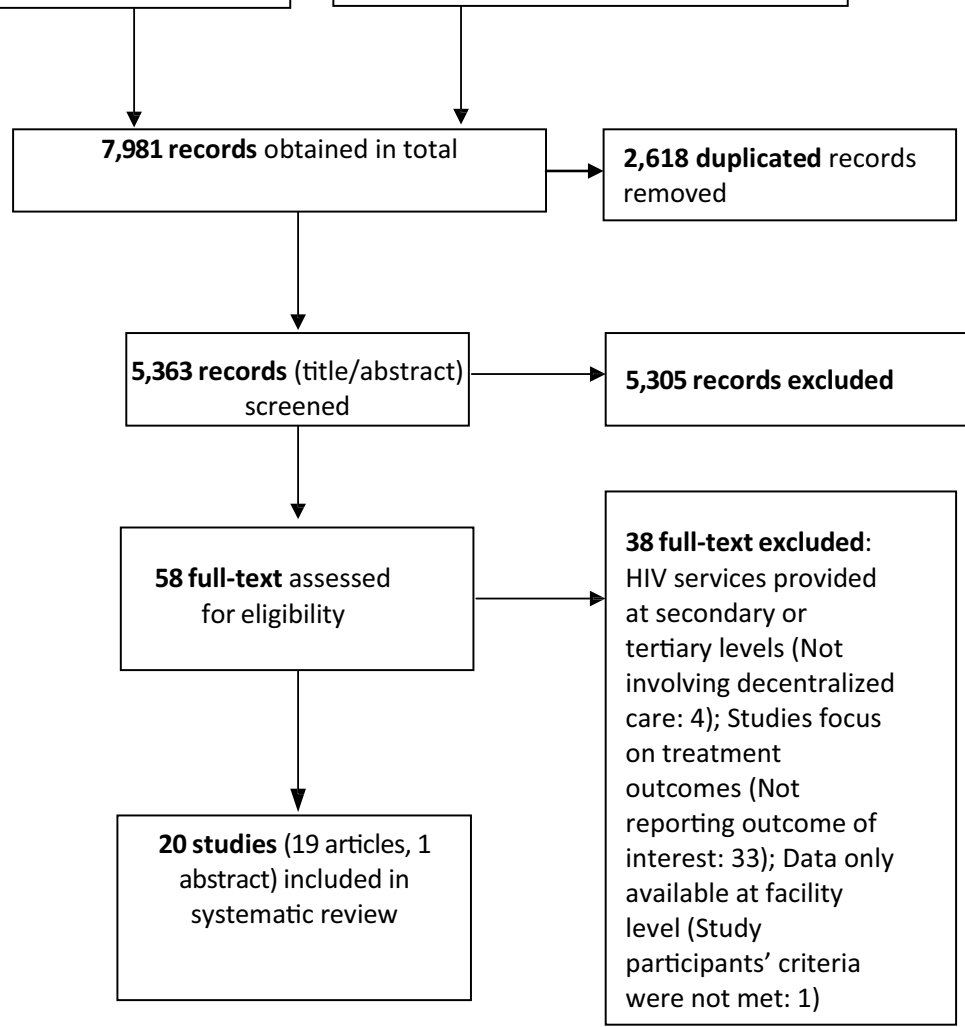

Fig. 1 Selection process of included studies

in rural settings (two in South Africa [20,21] and one in Rwanda [22]) reported $30-43 \%$ of patients had access to this service at 12 months follow-up although almost all (five of six) studies stated that VL (and CD4) was planned to be measured 6 monthly for all patients on ART. The ability of nursing staff to establish virological failure for timely referral and regimen switch was a concern as only $59 \%$ of patients who demonstrated persistently elevated $\mathrm{VL}$ in two consecutive VL monitoring tests were referred for further treatment intervention [18]. None of the included studies reported on-site VL or CD4 testing.

Among 12 studies that included data regarding virological assessment, only three studies reported the platform used for viral load testing (two studies with Nucli-Scens EasyQ HIV-1 and one study with a generic HIV VL platform-Biocentric) and none of these three studies discussed the blood sample used for VL testing (plasma or dried blood spot). None of the other nine studies reported how and where virological and/or immunological monitoring for patients on treatment was conducted.

\section{Clinical and immunological monitoring}

The majority (15 of 20) of included studies reported the use of WHO clinical staging to assess and monitor patients' responses to treatment (Table 2). Only three studies specifically provided data regarding the proportion of patients who received clinical monitoring through decentralized HIV treatment programs. The other 12 studies did not provide sufficient data for calculation of the coverage of clinical monitoring at decentralized settings.

One study [18] conducted in urban SA assessed the adherence of nursing staff at a primary health care clinic to national guidelines regarding monitoring and follow-up of HIV patients on ART. In this retrospective, cross-sectional study the authors randomly selected and assessed medical records of 488 patients attending the clinic from June 2011 to June 2012 and reported $84 \%(412 / 488)$ and $78 \%(381 / 488)$ patients with clinical monitoring data available by June 2011 and June 2012 respectively. 


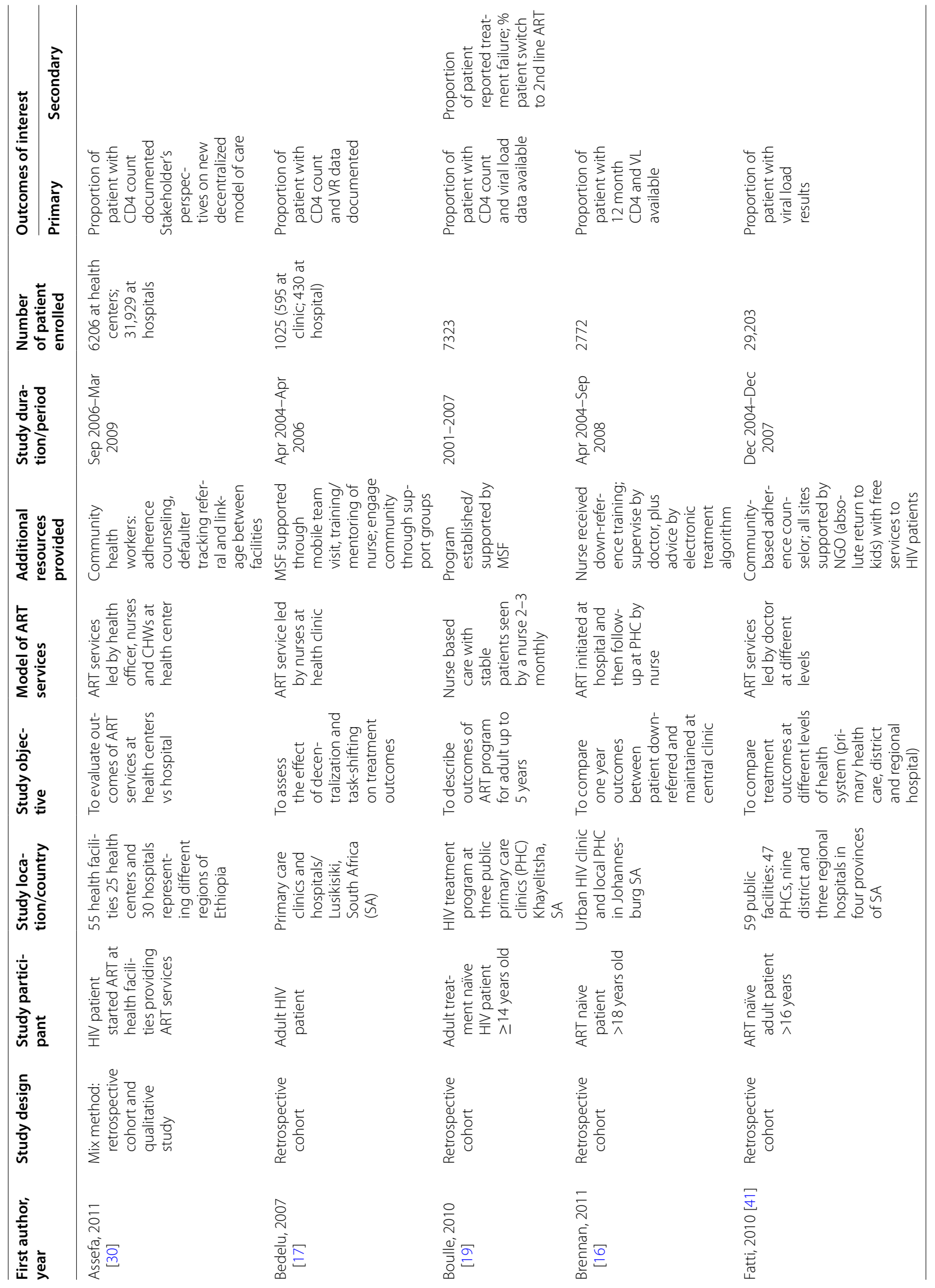




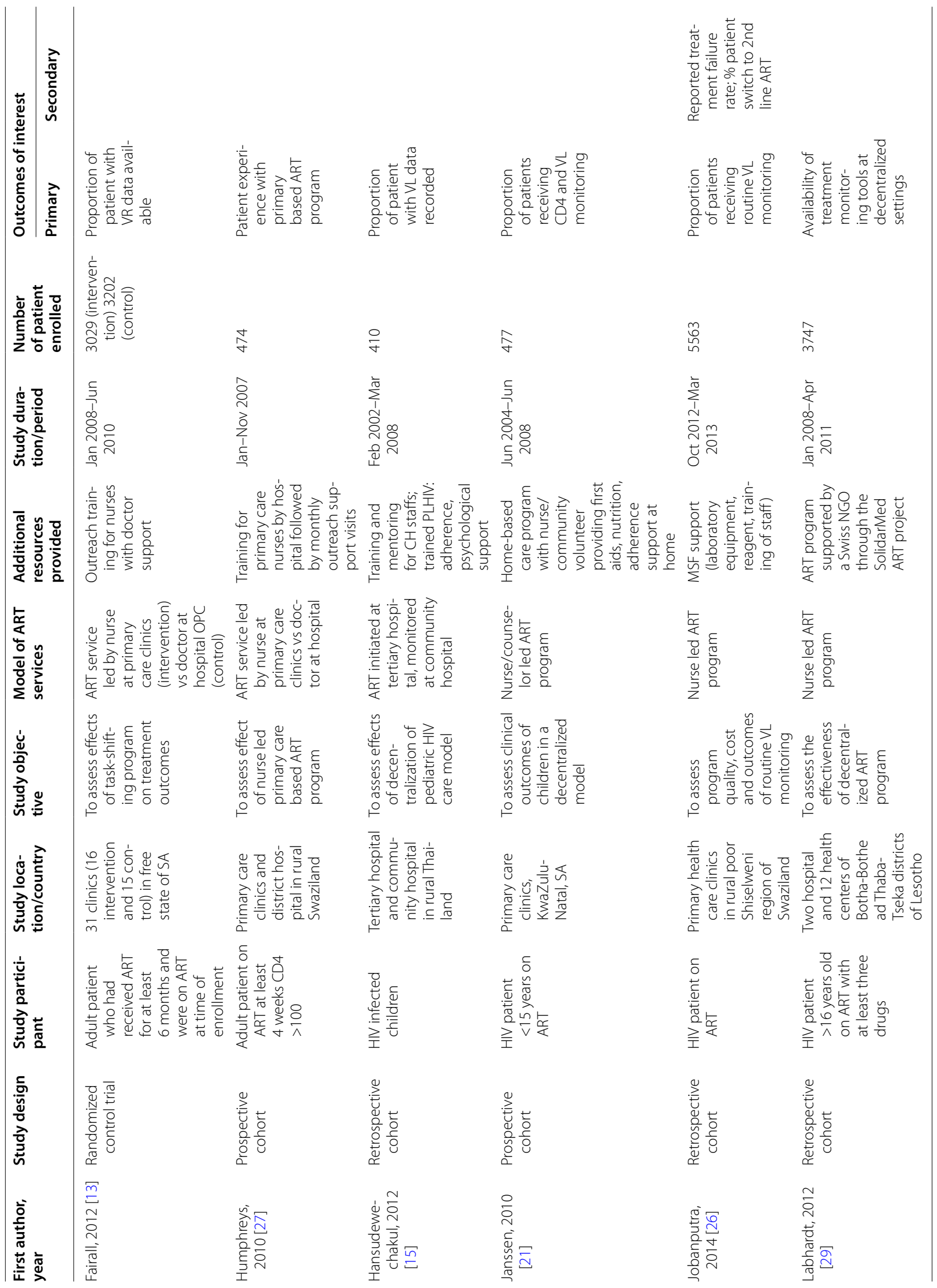




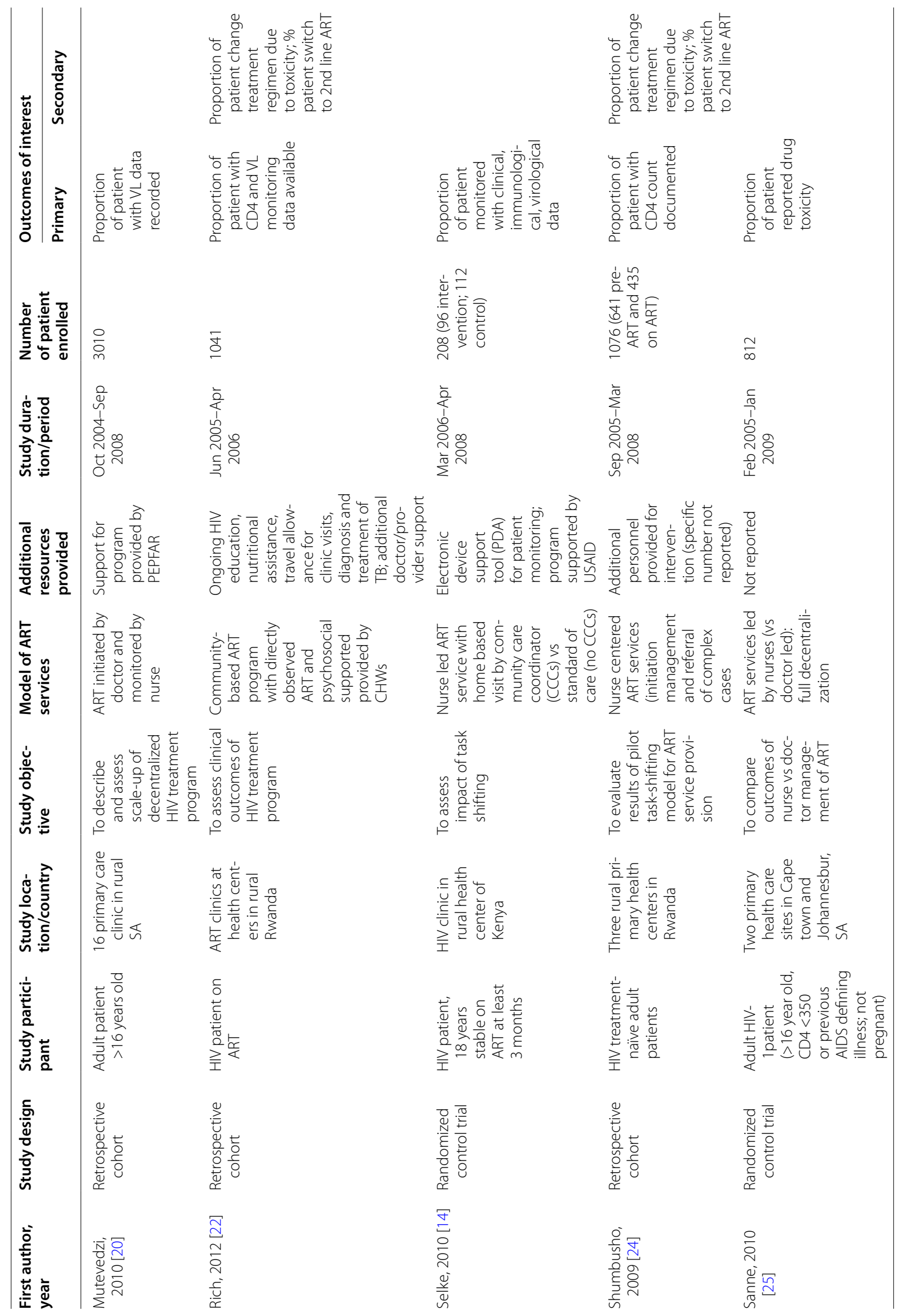




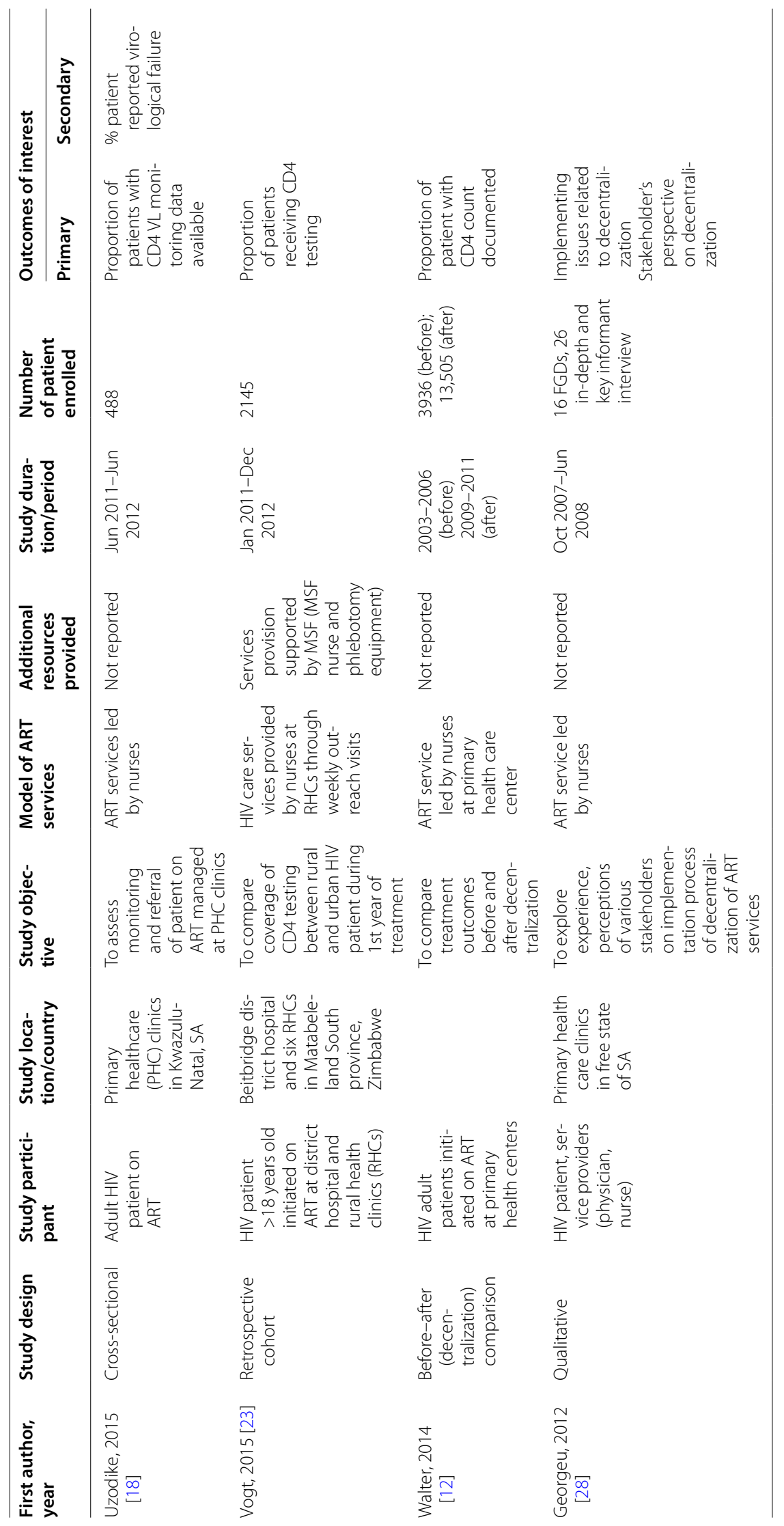




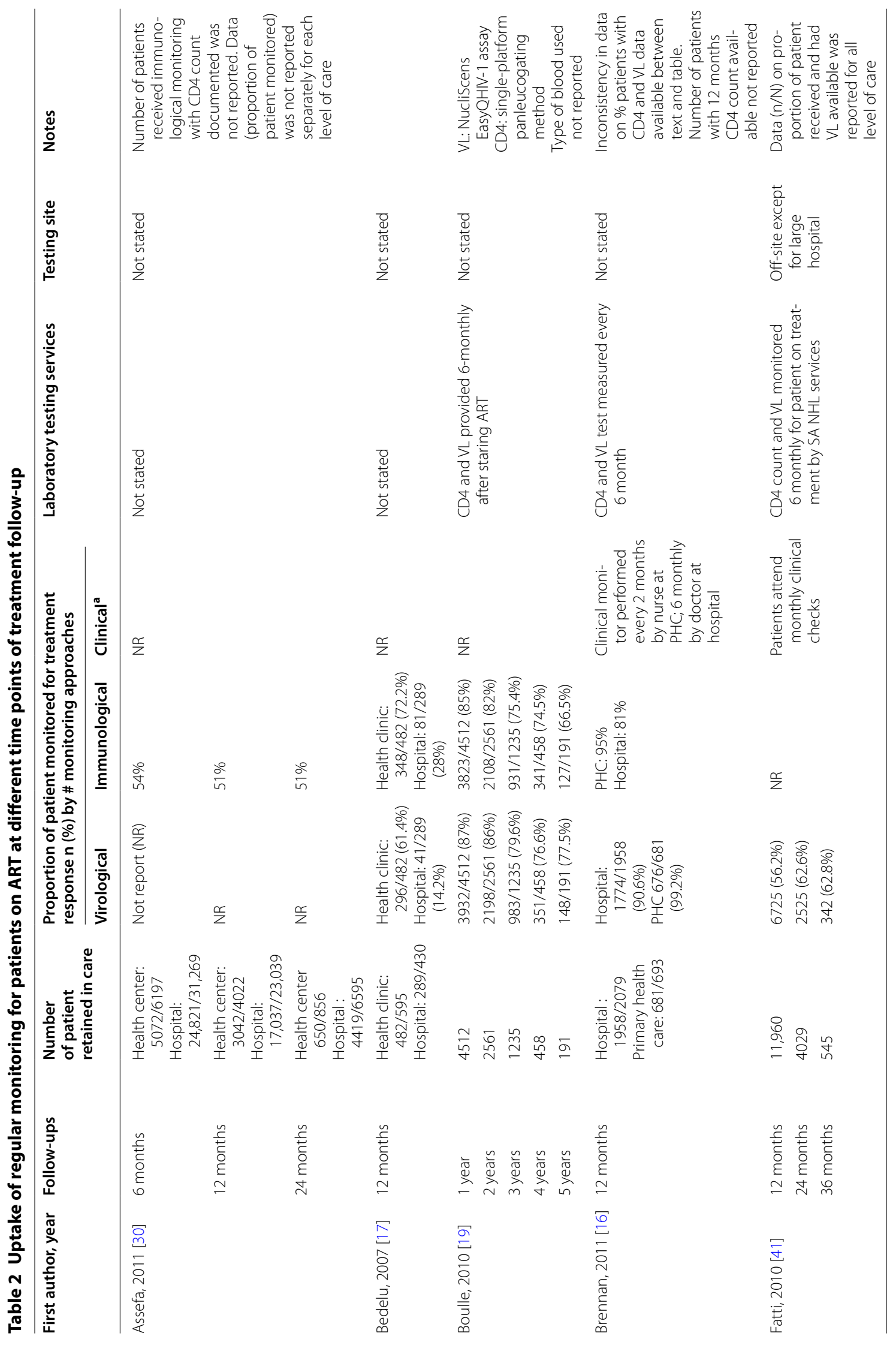




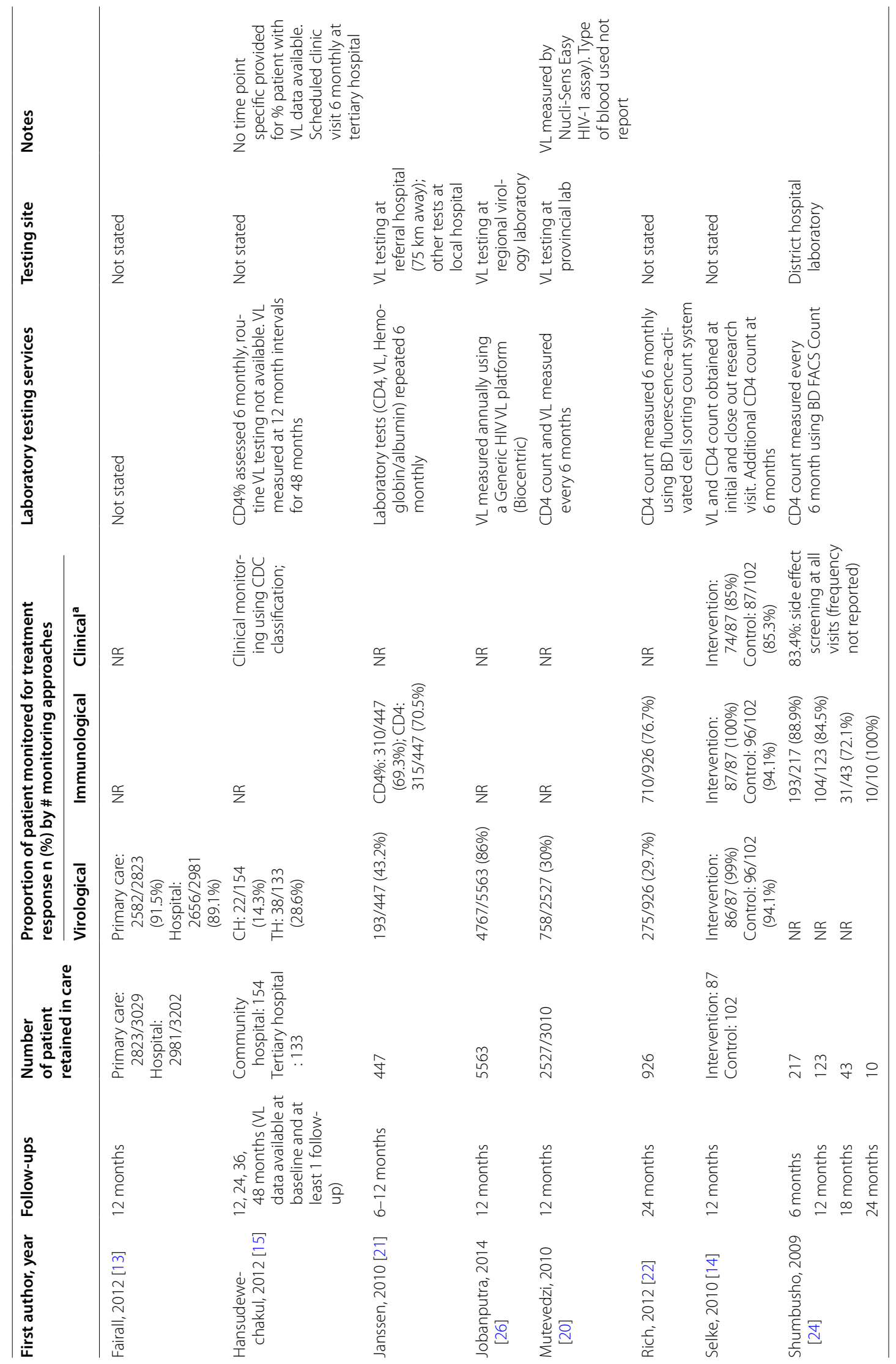




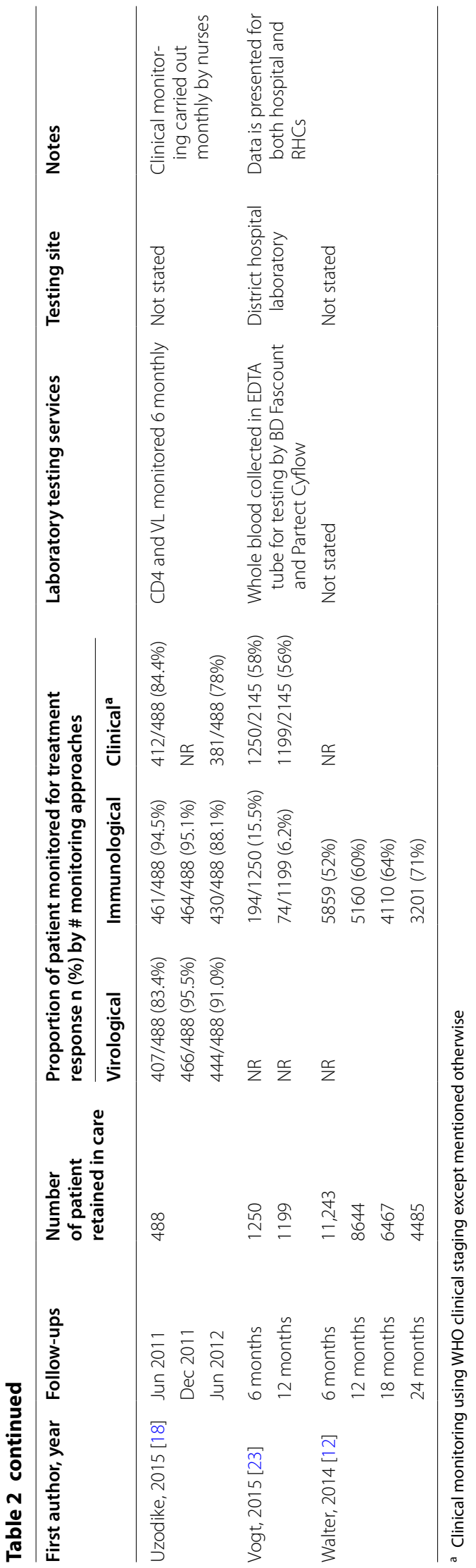


Another study [14] aimed to evaluate clinical outcomes of patients enrolled in a community-based HIV care program delivered by PLWHs (intervention group) as compared to patients receiving standard, clinic-based care (control group). The reported proportions of patients monitored clinically at 12 months follow-up were identical for both groups, $85 \%$ (74/87) for the control group and $85.3 \%$ (87/102) for the intervention group. One study [23] reported a lower level of clinical monitoring coverage with 1250 (58\%) and 1199 (56\%) out of 2145 patients initiated on ART receiving clinical assessments at 6 and 12 months follow-up, respectively.

Among 11 studies with patient follow-up periods from 6 to 24 months, the reported proportion of patients with a CD4 count measurement with data recorded at 6-12 month intervals ranged from 6 to $100 \%$. One study [19] with follow-up data of up to five years reported that $67 \%(127 / 191)$ to $85 \%(3823 / 4512)$ of patients had their CD4 count measured, and $78 \%(148 / 191)$ to $87 \%$ (3932/4512) of patients having their VL measured, at 12 month intervals. Data from this study showed that the proportion of patients receiving immunological and virological monitoring decreased over time, although the total number of patients in care also reduced by $96 \%$ after 5 years on treatment (from 4512 after 12 months to 191 after 5 years follow-up).

Two studies provided data that compared coverage of immunological monitoring between decentralized and centralized HIV care sites and both studies reported a higher uptake of services in the decentralized model. One study [17] reported $72 \%$ (348/482) of patients attending rural primary health care clinics versus $28 \%(81 / 289)$ attending a hospital had their CD4 count determined after 12 months of treatment. The authors reported that ART services provided at primary clinics were supported by Médecins Sans Frontières (MSF) with involvement of peer support groups to track defaulters, provide adherence support, advocate for better drug supply and monitoring of HIV program whereas no such supports were provided to patients receiving care at hospital. The second study [16] reported 95\% of down-referred (decentralized) patients $(n=693)$ versus $81 \%$ of centralized patients $(\mathrm{n}=2079)$ had a CD4 count (and VL) available at 12 months but the information on treatment monitoring procedure (platform used for VL testing, type of blood sample used and place where VL testing performed) was not presented.

One study [23] aimed to assess the coverage of immunological monitoring between HIV patients living in a semi-urban district in Zimbabwe and reported only 21 and $8 \%$ of urban $(n=1545)$, and 2 and $1 \%$ of rural patients $(n=600)$ had received CD4 testing at 6 and 12 months follow-up, respectively. The authors reported that CD4 testing at rural health centers was usually restricted to the day of outreach visits when outreach staff collected blood samples in Ethylenediaminetetraacetic Acid (EDTA) tubes and brought the blood back to the district hospital for testing within $24 \mathrm{~h}$. Limited capacity for specimen transportation within local health systems was noted as the main reason for the differences in access to CD4 testing between rural and urban patients.

\section{Toxicity monitoring}

No study reported the proportion of patients receiving laboratory monitoring for ARV drug toxicity at scheduled monitoring visits in program settings. The proportion of patients who changed their initial regimen (drug substitution, not considered as switching to second line ART) due to drug toxicity was reported as ranging from 5\% (161/3029) [13] to $29 \%(304 / 1040)$ [22]. One retrospective cohort study [24] reported $83.4 \%$ of all patients were screened for side-effects at all visits but the frequency of visits was not stated. A randomized controlled trial [25] reported 17\% (68/404) and 16\% (66/408) toxicity failure in patient groups managed by a nurse and by a doctor at primary care settings, respectively.

\section{Secondary outcomes}

Three studies reported the proportion of patients with virological failure and four studies reported the proportion of patients who switched from 1st to 2nd line ART. The reported proportion of patients with treatment failure ranged from $14 \%(n=4512)$ [19] to $49 \%(n=488)$ [18] and the proportion of patients starting 2 nd line treatment was from $0.5 \%(\mathrm{n}=1040)$ [22] during 24 months follow-up to $12.2 \%(n=4512)$ [19] at 60 months on treatment. One study assessed the outcomes of routine VL monitoring of ART programs through a decentralized network of 22 primary care clinics and three reference facilities in Zimbabwe. These investigators reported 17\% (551/3242) of VL tests had detectable HIV (>1000 copies/ $\mu \mathrm{L}$ ) and among 288 patients with an initial detectable VL result, 78 patients $(27 \%)$ did not receive adherence counseling, $86(30 \%)$ patients had no follow-up VL, and 15 patients $(5.2 \%)$ patients were switched to 2 nd line treatment, among whom four patients were switched based on a single detectable VL result [26].

\section{Factors that influence the implementation and feasibility of decentralization}

Data from included studies suggest that patients were supportive of decentralization of HIV treatment and care as it could help to improve their access to care (Table 3). One study [27] reported 96\% (29/31) of patients interviewed were 'very satisfied' or 'satisfied' with HIV 


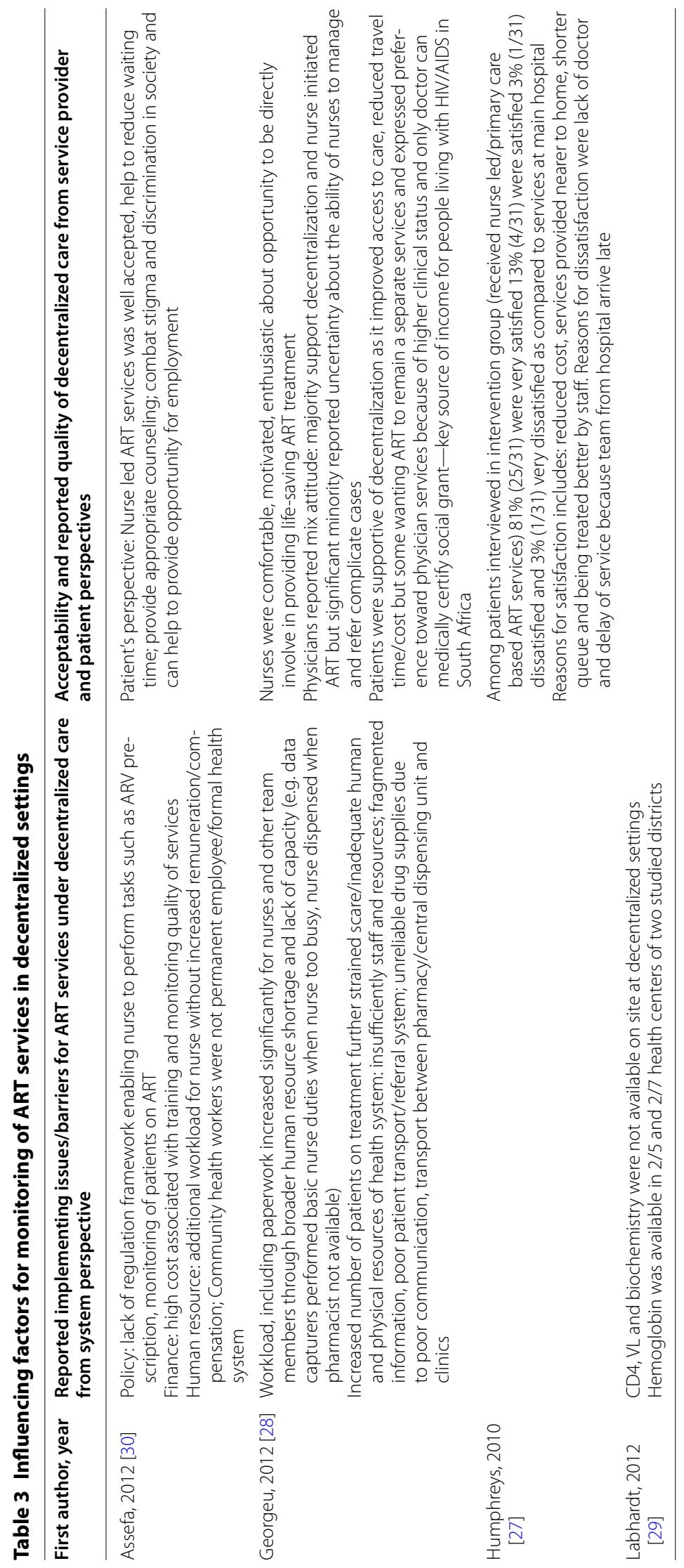


treatment services provided by nurses, and the main reasons for this included reduced cost, receiving services near home and shorter queue, and being treated better by staff. Health professionals also reported positive responses: nurses were comfortable, motivated, enthusiastic about the opportunity to be directly involved in providing life-saving treatment; physicians supported decentralization and nurse-led ART initiation as it could help increase ART coverage, but expressed uncertainty about the ability of nurses to manage and refer complicated cases [28].

A number of system factors that could hinder the implementation and scale-up of decentralization in lowresource settings were identified and discussed. These factors include: (i) Limited resources available for treatment monitoring services (ii) Lack of a policy framework which allows non-physician staff (nurses) to initiate HIV treatment; (iii) increased workload (clinical and administrative) for nurses without commensurate remuneration; (iv) unreliable antiretroviral drug supplies due to poor communication, inadequate transport between pharmacy/central dispensing unit and clinics; and (v) high costs associated with health worker training and monitoring of service quality $[29,30]$.

\section{Discussion}

\section{Why treatment monitoring is important to achieve the 90-90-90 goal}

Monitoring of patients on antiretroviral treatment (ART), especially in the context of rapid scale up of ART coverage in high HIV burden and low-resource settings through different models of services delivery including decentralization, is one of the most important elements to ensure effectiveness and sustainability of any HIV treatment and care program. The "90-90-90" goal aims at having $90 \%$ of HIV positive people knowing their infection status; $90 \%$ of those people receiving ART, and $90 \%$ of those on ART with virologic suppression, and is considered a universal target needed to effectively control and ultimately end the global HIV epidemic. There are two key milestones that need to be achieved to make the last "90" target a reality. First, the majority (>90\%) of patients on ART must have access to appropriate and timely ART monitoring: 12-monthly VL assessment or 6-monthly clinical assessment and CD4 count if VL is not available. Second, effective treatment and well-functioning patient support systems including adherence coaching must be in place to achieve a majority $(>90 \%)$ of patients on ART with sustained viral suppression.

\section{VL monitoring}

WHO has recommended VL testing as the preferred method for monitoring the responses of patients on ART
[8] and it has been suggested that in order to achieve the "90-90-90" goal, viral load monitoring should and can become the standard of care in LMICs with high HIV prevalence [31]. There is ample evidence showing that routine VL monitoring can provide an early and more accurate diagnosis of treatment failure when compared to clinical and immunological monitoring [32, 33], but evidence regarding the value of VL monitoring as compared to immunological monitoring in reducing mortality among patients on ART has been mixed [34-38]. Availability of, and access to, VL testing is still limited due to the requirements of expensive laboratory equipment, complex sample collection and processing, and the need for highly trained personnel [39]. A recent WHO survey on availability and use of HIV diagnostics in LMICs found that the overall coverage of VL testing among patients on ART from 94 countries was $23 \%$ [40]. In our review, a wide range of VL monitoring coverage was observed. Although we found a median VL monitoring coverage of $86 \%$ at 12 months follow-up, this level of coverage may not well reflect regular VL monitoring practice under decentralized HIV care models in LMICs. Among eight studies which reported VL monitoring rate of greater than $50 \%$ among patients on ART, five studies reported results of decentralized HIV programs supported by external donors such as MSF [17, 19, 26], Absolute Return for Kids [41], U.S. Agency for International Development (USAID) [14], and two studies reported results of randomized trials in which VL testing was part of the funded studies in well-resourced settings $[13,14]$. In addition, existing evidence suggests that the longer a patient is on ART the lower the rate of receiving regular VL test. Only three studies evaluated the longterm (more than 24 months) follow-up of VL coverage $[15,19,41]$. Thus, further research is needed to examine patient retention and treatment monitoring practices with long-term follow-up, particularly in rural settings.

\section{Clinical and immunological monitoring}

At decentralized primary care levels in LMICs, clinical and $\mathrm{CD} 4$ count monitoring remains a viable option to monitor treatment responses in settings where $\mathrm{VL}$ testing is not available. In our review, limited data were available to assess the feasibility and coverage of clinical and immunological monitoring in a decentralized model of HIV care, as only two studies provided data on the actual proportion of patients who received both clinical and CD4 monitoring. Of note, these are the two studies designed to assess treatment monitoring practice in two different settings, providing a contrasting picture of the coverage of treatment monitoring services. Differences in treatment monitoring coverage between these ART programs could be explained by study settings and service 
delivery models; one in an urban, well staffed HIV clinics with clinical staff on call and easy access to laboratory testing [18] while the other was in rural areas with ART service provided by outreach teams and with long distances for sample delivery from clinics to a laboratory facility located at a district hospital [23]. This finding highlights an important potential gap in existing knowledge. It has implications related to the implementation of treatment monitoring in future decentralized ART programs, particularly in rural, resource-constraint settings, as when only a minority of patients is engaged in an ART program where they receive regular monitoring, an increase in treatment failure and drug resistance can be foreseen.

\section{Drug toxicity monitoring}

Limited data were available to assess the feasibility of drug toxicity monitoring for patients on ART in decentralized settings. None of the included studies reported the proportion of patients on ART who received laboratory-based drug toxicity monitoring, but one RCT showed that the proportion of patients reporting toxic drug effects (defined as adverse events that required treatment interruption for $>42$ days) during the study period was higher than the total virological failure rate among patients on ART [25]. This finding is in line with results from other studies suggesting that drug toxicity is the most common reason for changing initial treatment regimen $[42,43]$. The WHO guidelines emphasize that laboratory monitoring is not required for treatment initiation. However, there are major toxicities associated with ARV drugs that should be monitored in all patients on treatment. The basic monitoring for potential toxicity of drugs such as tenofovir, zidovudine and nevirapine require laboratory assessment of renal function, hemoglobin, and liver enzymes, respectively. Without the availability of, and access to, these basic tests, monitoring for ART toxicity cannot be performed, and could compromise the long-term effectiveness and sustainability of the ART program. Researches have showed that HIV patients on ART who have regimen substitution due to drug toxicity/drug related adverse reactions were at higher risk of loss to follow-up [44, 45] which may partly explain the significant reduction in number of patients in care after five years of follow-up reported from study included in this review [19].

\section{Barriers related to treatment monitoring and evaluation of treatment monitoring under decentralization Technological constraints}

From a technology perspective, in the absence of point of care $(\mathrm{POC})$ testing, access to laboratory monitoring for patients on ART under a decentralized model of care in low-resource settings will likely be limited. For VL monitoring; until the arrival of a true point of care VL test, the feasibility of VL monitoring for patients received ART at the primary clinic level will depend on system capacity to collect and process a blood sample, transport the sample to a central laboratory for testing and return the result in a timely manner. For immunological monitoring, there are CD4 POC technologies available that can be operated in decentralized settings and produce reliable results for treatment monitoring [46]; their use has been shown to improve access to this alternative monitoring method and increases patient retention along the HIV treatment cascade compared to conventional laboratory testing [47].

\section{Human resource constraints}

A lack of trained medical doctors for initiation and management of patients on ART has been identified as a major barrier for scaling up of ART programs [48, 49]. Task-shifting of HIV services from physician to non-physician carers has been introduced to overcome this challenge $[4,13]$. From a treatment monitoring perspective, however, task-shifting does not come without challenges. Findings from our review suggest that increased clinical and administrative responsibilities associated with provision of nurse-led ART services could further burden the already-limited personnel at primary health care level. Primary health care staff reported their reluctance to put more PLWHA on treatment because of concern over their capacity to manage the burden of an increasing number of patients on ART. The quality of treatment monitoring could also be a concern as nursing staff were unable to identify and refer all cases of treatment failure at decentralized settings for regimen change, even with the availability of two consecutive VL monitoring results indicating virological failure. The lower than expected rate of patients initiated on second line ART may represent an appropriate strategy to optimize adherence before switching therapy but it may also indicate clinicians' lack of confidence regarding interpretation of VL results and second line treatment. The introduction of any new assay into a clinical setting requires education of the clinician in its interpretation; this is especially the case with a complicated tool such as a VL test. On the other hand, early switching to second line ART after a single detectable VL test without appropriately addressing non-adherence issues would potentially result in the unnecessary initiation of second line ART and, without addressing poor adherence would lead to suboptimal second-line outcomes $[50,51]$. This is an important issue of concern particularly in settings where treatment options are limited and second and third-line regimens are costly. 


\section{Recommendations}

In LMICs the challenge of limited coverage of, and access to, treatment monitoring services that is associated with decentralization of HIV treatment and care often lies within the health care system; therefore a comprehensive strategy to improve the practice of treatment monitoring should be considered from a health system strengthening perspective (Fig. 2).

In terms of service delivery, treatment and treatment monitoring services should ideally be delivered close to where the patient lives, with appropriate diagnostic technology and human resource availability at the primary health care level. The development and implementation of POC technologies to provide immunological and virological monitoring are critically important to ensure appropriate treatment monitoring, particularly with further scale up of HIV treatment services in decentralized settings.

The impact of future studies towards improving the implementation of decentralized care would benefit from the inclusion of some standardized targets and outcomes in published reports. In the absence of clearly defined indicators and targets, the assessment and appraisal of coverage and quality of treatment monitoring services continues to be a challenge. Given the momentum in scaling up ART and towards achieving the 90-90-90 target, there is a need for standardized measures that can be used in many upcoming researches reporting global progress towards this ambitious goal. The development and adoption of a specific set of processes and target indicators regarding treatment monitoring could help to align the reporting system within different levels of health services provision, improve the timeliness of reporting results, and ensure that appropriate action is taken when results support particular interventions (e.g. adherence counseling).

Lastly, from governance and financing perspectives, it is obvious that if the ambitious "90-90-90" goal is to be achieved in 2020, the importance of treatment monitoring must be emphasized equally with the importance of treatment coverage. Substantial resources are required to ensure appropriate treatment monitoring for all people on ART. Critical to success is the assessment of system
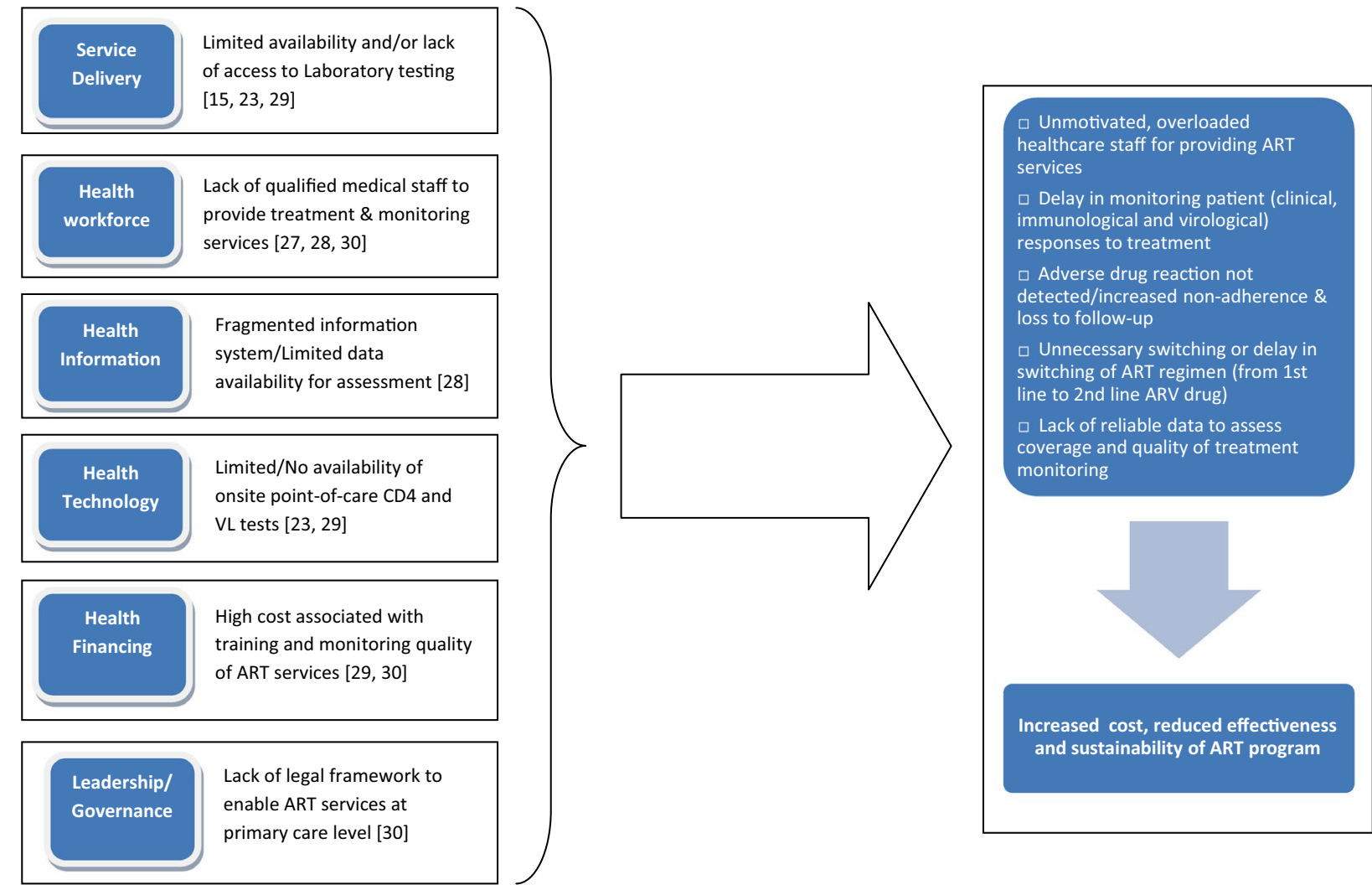

Fig. 2 Health system challenges impacting access to ART monitoring (clinical/immunological, viral load and drug toxicity) based on data from studies included in the review 
capacity, particularly human resources and health technology in delivering treatment monitoring. This must be conducted as an integrated component of the decisionmaking process in order to identify the optimal strategy to increase high quality coverage of HIV treatment and care services in any given specific setting. Expansion of ART coverage without considering system capacity for the provision of appropriate treatment monitoring to all patients will inevitably lead to more treatment failures and increased development of drug resistance, with resulting public health costs to address these problems. Therefore, the recommendation of WHO that lack of access to, or availability of, laboratory monitoring should not be a barrier in initiating patients on treatment may need to be revisited, as the closer we get to the second " 90 " goal of having $90 \%$ people diagnosed with HIV on treatment, the higher the importance of assuring that those patients who are on treatment are also appropriately monitored, such that the last " 90 " goal of having $90 \%$ people on treatment with viral suppression can be achieved.

\section{Limitation}

This review has some limitations that should be taken into account when interpreting the findings. Here, we identified only two studies that aimed to assess the monitoring and management of HIV patients. This paucity of data results in challenges regarding data interpretation and meant that we were unable to analyze and discuss differences in coverage of treatment monitoring services as well as quality of the services. Lack of information and data from unpublished government and program reports and studies published in non-English language may contribute to limited data availability. Moreover, limited data from studies conducted in SSA countries has made it difficult to generalize the findings outside the sub-Saharan African context.

\section{Conclusions}

The findings of this review suggest that there are potential major gaps in coverage and quality of treatment monitoring services for HIV patients on ART. Further studies particularly from non-SSA countries with longer-term of follow up are in need to assess the feasibility of treatment monitoring in the context of decentralization HIV treatment and care in LMICs. Significant investment in POC testing and, improving quality of and training for nursing staff to effectively manage patients on ART is required to improve quality of HIV treatment and care services. The development of a set of target program indicators for treatment monitoring is necessary to reinforce the importance of treatment monitoring in the HIV continuum of care toward achievement of the 90-90-90 goal by the year 2020.

\section{Additional file}

Additional file 1: Annex S1. Search strategy: Decentralization of HIV treatment and care in low and middle income countries.

\section{Abbreviations}

ART: antiretroviral therapy; PLWH: people living with HIV/AIDS; LMICs: Iow and middle income countries; SSA: sub-Saharan Africa; SA: South Africa; VL: viral load; RCT: randomized control trial; POC: point of care.

\section{Authors' contributions}

MP, SL, DA, SC developed review protocol. LR, MP performed literature search. $M P, B P$ performed data extraction and drafted the manuscript. LR, BP, DA, SC, $S L$ reviewed and commented on initial and final drafts of the manuscript. All authors read and approved the final manuscript.

\section{Author details}

${ }^{1}$ Burnet Institute, 85 Commercial Road, Melbourne, VIC 3004, Australia. ${ }^{2}$ Department of Epidemiology and Preventive Medicine, Faculty of Medicine Nursing and Health Science, Monash University, Melbourne, Australia. ${ }^{3}$ The Alfred Hospital, The lan Potter Library, Melbourne, VIC, Australia. ${ }^{4}$ Department of Microbiology, Faculty of Medicine Nursing and Health Science, Monash University, Melbourne, Australia. ${ }^{5}$ Department of Infectious Diseases, The Alfred Hospital Melbourne, Melbourne, Australia. ${ }^{6}$ Monash School of Medicine, Faculty of Medicine Nursing and Health Science, Monash University, Melbourne, Australia. ${ }^{7}$ International Centre for Reproductive Health, Department of Obstetrics and Gynecology, Faculty of Medicine and Health Sciences, Ghent University, Ghent, Belgium.

\section{Acknowledgements}

The authors gratefully acknowledge the contribution to this work of the Victorian Operational Infrastructure Support Program received by the Burnet Institute. We thank Peter Higgs at the Burnet Institute for his valuable comments on the draft of the manuscript.

\section{Competing interests}

The authors declare that they have no competing interests.

\section{Availability of data and materials}

All data generated or analysed during this study are included in this published article.

\section{Funding}

Funding was provided by the National Health and Medical Research Council of Australia (NHMRC) (Project Grant GNT 1063725; Career Development Fellowship to S. Luchters, Principal Research Fellowship to S. Crowe, and Infrastructure for Research Institutes Support Scheme Grant). Minh Pham received support via an International Postgraduate Research Scholarship (IPRS) from the Commonwealth of Australia and the Victorian International Research Scholarship (VIRS) from State Government of Victoria, Australia.

Received: 10 October 2016 Accepted: 6 January 2017

Published online: 19 January 2017

\section{References}

1. Sidibe M, Zuniga JM, Montaner J. Leveraging HIV treatment to end AIDS, stop new HIV infections, and avoid the cost of inaction. Clin Infect Dis. 2014;59(Suppl 1):S3-6.

2. UNAIDS. "15 by 15" a global target achieved. 2015. Joint United Nations Programme on HIV/AIDS: Geneva, 2015.

3. Scanlon ML, Vreeman RC. Current strategies for improving access and adherence to antiretroviral therapies in resource-limited settings. HIV AIDS. 2013;5:1-17.

4. Kredo T, et al. Task shifting from doctors to non-doctors for initiation and maintenance of antiretroviral therapy. Cochrane Database Syst Rev. 2014;7:CD007331. 
5. Kredo T, et al. Decentralising HIV treatment in lower- and middle-income countries. Cochrane Database Syst Rev. 2013;6:CD009987.

6. Suthar AB, et al. Improving antiretroviral therapy scale-up and effectiveness through service integration and decentralization. Aids. 2014;28:5175-85.

7. WHO. Guideline on when to start antiretroviral therapy and on preexposure prophylaxis for HIV. Geneva: World Health Organization; 2015. p. 78.

8. WHO. Consolidated guidelines on the use of antiretroviral drugs for treating and preventing HIV infection recommendations for a public health approach. Geneva: World Health Organization; 2013. p. 272

9. Crowley T, Stellenberg EL. Integrating HIV care and treatment into primary healthcare: are clinics equipped? Afr J Prim Health Care Fam Med. 2014;6(1):E1-7.

10. Sawe FK, McIntyre JA. Monitoring HIV antiretroviral therapy in resourcelimited settings: time to avoid costly outcomes. Clin Infect Dis. 2009;49(3):463-5.

11. Moher $\mathrm{D}$, et al. Preferred reporting items for systematic reviews and meta-analyses: the PRISMA statement. PLoS Med. 2009;6(7):e1000097.

12. Walter J, et al. Treatment outcomes before and after the decentralization of art in an Urban setting in Mozambique. Top Antivir Med. 2014:22:555-6.

13. Fairall $\mathrm{L}$, et al. Task shifting of antiretroviral treatment from doctors to primary-care nurses in South Africa (STRETCH): a pragmatic, parallel, cluster-randomised trial. Lancet. 2012;380(9845):889-98.

14. Selke HM, et al. Task-shifting of antiretroviral delivery from health care workers to persons living with HIV/AIDS: clinical outcomes of a community-based program in Kenya. J Acquir Immune Defic Syndr JAIDS. 2010;55(4):483-90.

15. Hansudewechakul R, et al. Successful clinical outcomes following decentralization of tertiary paediatric HIV care to a community-based paediatric antiretroviral treatment network, Chiangrai, Thailand, 2002 to 2008. J Int AIDS Soc. 2012:15(2):17358.

16. Brennan AT, et al. Outcomes of stable HIV-positive patients downreferred from a doctor-managed antiretroviral therapy clinic to a nursemanaged primary health clinic for monitoring and treatment. AIDS. 2011;25(16):2027-36.

17. Bedelu $\mathrm{M}$, et al. Implementing antiretroviral therapy in rural communities: the Lusikisiki model of decentralized HIV/AIDS care. J Infect Dis. 2007;196(Suppl 3):S464-8.

18. Uzodike N, Ross A, Harbor O. Adherence by a primary healthcare clinic in KwaZulu-Natal to the national HIV guidelines. S Afr Fam Pract. 2015;57(3):198-202.

19. Boulle $A$, et al. Seven-year experience of a primary care antiretroviral treatment programme in Khayelitsha, South Africa. AIDS. 2010;24(4):563-72.

20. Mutevedzi PC, et al. Scale-up of a decentralized HIV treatment programme in rural KwaZulu-Natal, South Africa: does rapid expansion affect patient outcomes? Bull World Health Organ. 2010;88(8):593-600.

21. Janssen $\mathrm{N}$, et al. Successful paediatric HIV treatment in rural primary care in Africa. Arch Dis Child. 2010;95(6):414-21.

22. Rich ML, et al. Excellent clinical outcomes and high retention in care among adults in a community-based HIV treatment program in rural Rwanda. JAcquir Immune Defic Syndr JAIDS. 2012;59(3):e35-42.

23. Vogt F, et al. Access to CD4 testing for rural HIV patients: findings from a cohort study in Zimbabwe. PLoS ONE. 2015;10(6):e0129166.

24. Shumbusho F, et al. Task shifting for scale-up of HIV care: evaluation of nurse-centered antiretroviral treatment at rural health centers in Rwanda. PLoS Med. 2009;6(10):e1000163.

25. Sanne I, et al. Nurse versus doctor management of HIV-infected patients receiving antiretroviral therapy (CIPRA-SA): A randomised non-inferiority trial. Lancet. 2010;376(9734):33-40.

26. Jobanputra K, et al. Impact and programmatic implications of routine viral load monitoring in Swaziland. J Acquir Immune Defic Syndr. 2014;67(1):45-51.

27. Humphreys $C P$, et al. Nurse led, primary care based antiretroviral treatment versus hospital care: a controlled prospective study in Swaziland. BMC Health Serv Res. 2010;10:229.

28. Georgeu D, et al. Implementing nurse-initiated and managed antiretroviral treatment (NIMART) in South Africa: a qualitative process evaluation of the STRETCH trial. Implement Sci. 2012;7:66.
29. Labhardt ND, et al. Adoption of new HIV treatment guidelines and drug substitutions within first-line as a measure of quality of care in rural Lesotho: health centers and hospitals compared. Trop Med Int Health. 2012;17(10):1245-54

30. Assefa $Y$, et al. Effectiveness and acceptability of delivery of antiretroviral treatment in health centres by health officers and nurses in Ethiopia. J Health Serv Res Policy. 2012;17(1):24-9.

31. Lecher S, et al. Scale-up of HIV viral load monitoring — seven sub-Saharan African countries. MMWR Morb Mortal Wkly Rep. 2015;64(46):1287-90.

32. Essajee S, Kumarasamy N. Commentary: the monitoring of adults and children on antiretroviral therapy in the 2013 WHO consolidated ARV guidelines. AIDS. 2014;28(Suppl 2):S147-9.

33. Rutstein SE, et al. On the front line of HIV virological monitoring: barriers and facilitators from a provider perspective in resource-limited settings. AIDS Care. 2016;28(1):1-10.

34. Jourdain G, et al. Switching HIV treatment in adults based on CD4 count versus viral load monitoring: a randomized, non-inferiority trial in Thailand. PLoS Med. 2013;10(8):e1001494.

35. Mermin J, et al. Utility of routine viral load, CD4 cell count, and clinical monitoring among adults with HIV receiving antiretroviral therapy in Uganda: randomised trial. BMJ. 2011;343:d6792.

36. Laurent C, et al. Monitoring of HIV viral loads, CD4 cell counts, and clinical assessments versus clinical monitoring alone for antiretroviral therapy in rural district hospitals in Cameroon (Stratall ANRS 12110/ESTHER): a randomised non-inferiority trial. Lancet Infect Dis. 2011;11(11):825-33.

37. Estill J, et al. Monitoring of antiretroviral therapy and mortality in HIV programmes in Malawi, South Africa and Zambia: mathematical modelling study. PLoS ONE. 2013;8(2):e57611.

38. Shen Z, et al. Effects of CD4 cell counts and viral load testing on mortality rates in patients with HIV infection receiving antiretroviral treatment: an observational cohort study in rural southwest China. Clin Infect Dis. 2016;63:108-14.

39. Roberts T, et al. Scale-up of routine viral load testing in resource-poor settings: current and future implementation challenges. Clin Infect Dis. 2016;62:1043-8.

40. WHO. The availability and use of HIV diagnostics: a 2012/2013 WHO survey in low- and middle-income countries. Geneva: World Health Organization; 2014. p. 29

41. Fatti G, Grimwood A, Bock P. Better antiretroviral therapy outcomes at primary healthcare facilities: an evaluation of three tiers of ART services in four South African provinces. PLOS ONE. 2010;5(9):e12888.

42. Abaissa SG, et al. Adverse drug reactions associated with antiretroviral treatment among adult ethiopian patients in a tertiary hospital. Ethiop Med J. 2012;50(2):107-13.

43. Jima YT, Angamo MT, Wabe NT. Causes for antiretroviral regimen change among HIV/AIDS patients in Addis Ababa, Ethiopia. Tanzan J Health Res. 2013;15(1):11-8.

44. Berheto TM, Haile DB, Mohammed S. Predictors of loss to follow-up in patients living with HIV/AIDS after initiation of antiretroviral therapy. $\mathrm{N}$ Am J Med Sci. 2014;6(9):453-9.

45. Tupasi TE, et al. Factors associated with loss to follow-up during treatment for multidrug-resistant tuberculosis, the Philippines, 2012-2014. Emerg Infect Dis. 2016;22(3):491-502.

46. Scott $L E$, et al. A meta-analysis of the performance of the Pima CD4 for point of care testing. BMC Med. 2015;13:168.

47. Vojnov $L$, et al. POC CD4 testing improves linkage to HIV care and timeliness of ART initiation in a public health approach: a systematic review and meta-analysis. PLoS ONE. 2016;11(5):e0155256.

48. Kober K, Van Damme W. Scaling up access to antiretroviral treatment in southern Africa: who will do the job? Lancet. 2004;364(9428):103-7.

49. Mbewu AD. Changing history-closing the gap in AIDS treatment and prevention. Bull World Health Organ. 2004;82(6):400.

50. Johnston V, et al. Viral suppression following switch to second-line antiretroviral therapy: associations with nucleoside reverse transcriptase inhibitor resistance and subtherapeutic drug concentrations prior to switch. J Infect Dis. 2014:209(5):711-20.

51. Ramadhani $\mathrm{HO}$, et al. Association of first-line and second-line antiretroviral therapy adherence. Open Forum Infect Dis. 2014;1 (2):0fu079. 